\title{
Power Control in Cellular Radio Systems Subject to Constraints and Time Delays
}

\author{
Fredrik Gunnarsson, Jonas Blom and Fredrik Gustafsson \\ Department of Electrical Engineering \\ Linköpings universitet, SE-581 83 Linköping, Sweden \\ WWW: http://www.control.isy.liu.se \\ Email: fred@isy.liu.se, jb@isy.liu.se, \\ fredrik@isy.liu.se
}

April 16, 1999

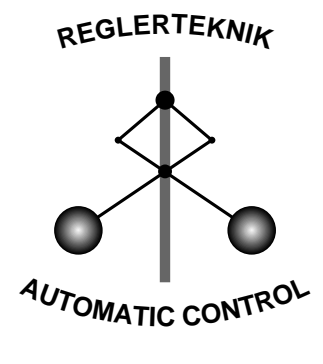

LINKÖPING

Report no.: LiTH-ISY-R-2144

Submitted to GLOBECOM'98, Sydney, Australia

Technical reports from the Automatic Control group in Linkping are available by anonymous ftp at the address ftp.control.isy.liu.se. This report is contained in the compressed postscript file 2144 .ps. Z. 



\begin{abstract}
Stability is a fundamental property desirable for any controlled system. We briefly review the root locus and describing function techniques, which are tools for stability analysis, and show how they can be applied to power control algorithms in cellular radio networks. The root locus method is used to find stability limits on controller parameters, and describing functions for predicting the presence of oscillations in the system. Thus these methods can be used to support the design phase, when deciding upon the appropriate controller parameters. These tools are demonstrated for various control algorithms and when different smoothing filters are applied. The analysis reveals that the Distributed Power Control (DPC) algorithm, which works fine under ideal circumstances, yields an unstable system when subject to a small time delay. Furthermore, it is concluded that the performance with respect to stability is better when the measurements are averaged by exponential forgetting filter than by the moving average filter.
\end{abstract}

Keywords: Cellular radio systems; Power Control Algorithms; Local loop analysis; Time delays; Root locus; Describing functions 


\section{Introduction}

Due to the rapid expansion of the wireless mobile market, and the need for wideband multimedia services, the available bandwidth has to be better utilized. Several transmitter power control algorithms have been proposed to improve the capacity. Most schemes strive to balance the carrier-to-interference ratios $(\mathrm{C} / \mathrm{I})$ on each channel such that every mobile or base station achieve the same $\mathrm{C} / \mathrm{I}$ $[17]$.

To avoid extensive control signaling in the network, it is desirable to use distributed algorithms, where the transmitter powers are locally controlled based on local measurements (e.g. C/I). Such distributed algorithms have previously been studied in $[16,9,8,1,14,11,2,13]$.

These algorithms perform well in rather ideal cases, but in real systems there are a number of effects that hamper the performance. Firstly, the possible output powers of the transmitters are limited due to physical constraints. Schemes that consider constrained powers are studied in [2, 10, 15]. Additional complexity arise when considering the fact that the transmitter powers are not only limited but also quantized and thus only a discrete set of power levels are allowed. These problems are addressed in [3]. All these constraints are in essence non-linear components in the power control loop.

Secondly, measuring and control signaling take time, which result in time delays in the network. It takes some time to measure and report the measurements to the algorithm, resulting in a time delay of $n_{m}$ samples. In addition we have a time delay of $n_{p}$ samples due to the time before the computed power is actually used in the transmitter and experienced by others. The total delay is $n=n_{p}+n_{m}$. Time delays in the power controlled systems have been left out or neglected in most previous work.

Thirdly, in order to reduce the effects of inaccurate measurements, various filters are used to obtain smooth estimates. Moving average and exponential forgetting filters are two common examples.

Finally, the choice of an adequate quality measure and the availability of measurements are important issues. The common assumption is that the transmission quality is depending only on the $\mathrm{C} / \mathrm{I}$, which is assumed to be measured or estimated. The analysis in this paper is based on such a simplifying assumption. However, in real systems the situation is more complex, and it is further discussed in $[5,7]$.

Hence we can describe the surrounding environment, as seen by the decentralized power controller, as in Figure 1.

The effects of time delays and constraints, with respect to stability, will be studied in this work. Techniques from the field of automatic control will be used to analyze the effects on stability and oscillatory behavior of algorithms, but first we will review some basic definitions and state the problems formally. 


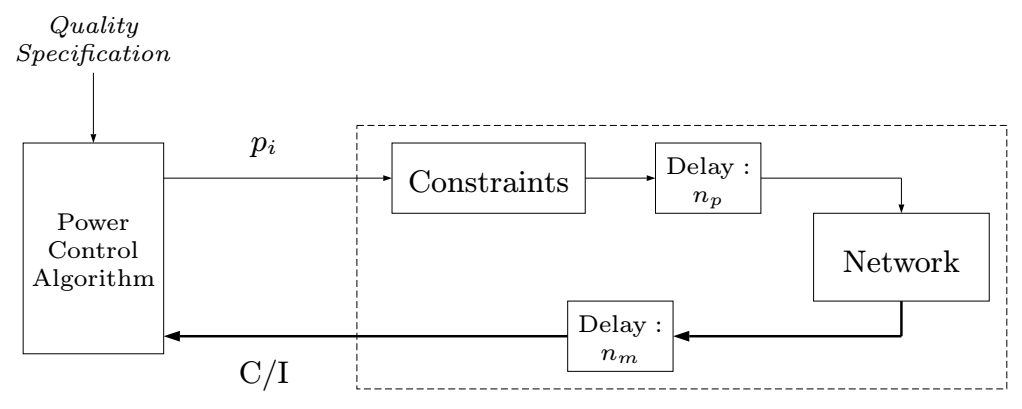

Environment

Figure 1: The surrounding Environment as seen by a decentralized controller, when considering time delays and constraints. The Network block incorporates the effects caused by the radio channel, such as power gain, noise and interfering transmitters.

\section{System Model}

\subsection{Mobile Communication Network}

Signal gains and power levels can be expressed using different scales. Logarithmic (e.g. $d B$ or $d B m$ ) or linear are often used. To avoid confusion we will employ the convention of indicating linearly scaled values with a bar. Thus $\bar{g}_{i j}$ is a value in linear scale and $g_{i j}$ the corresponding value in logarithmic scale.

Focus on the mobile stations and base stations using a specific channel and assign numbers such that mobile station $i$ is connected to base station $i$. The signal gain from base station $j$ to mobile station $i$ is denoted by $\bar{g}_{i j}$.

Assume that the $m$ mobile stations are transmitting using the powers $\bar{p}_{i}(t), i=$ $1, \ldots, m$. The corresponding connected base stations will experience a desired carrier signal $\bar{C}_{i}(t)=\bar{g}_{i i}(t) \bar{p}_{i}(t)$ and an interference plus noise $\bar{I}_{i}(t)$, which is the sum of the signals from all other mobiles and the thermal noise $\bar{\nu}_{i}(t)$. Thus we can define the carrier-to-interference ratio at base station $i$ as

$$
\bar{\gamma}_{i}(t)=\frac{\bar{C}_{i}(t)}{\bar{I}_{i}(t)}=\frac{\bar{g}_{i i}(t) \bar{p}_{i}(t)}{\sum_{j \neq i} \bar{g}_{i j}(t) \bar{p}_{j}(t)+\bar{\nu}_{i}(t)} .
$$

We will assume that the Quality of Service (QoS) is depending only on the C/I, and is acceptable iff $\mathrm{C} / \mathrm{I}$ is above a certain threshold

$$
\bar{\gamma}_{i}(t) \geq \bar{\gamma}^{*}, i=1, \ldots, m
$$




\subsection{Power Control Algorithms}

One of the most discussed distributed algorithms is the Distributed Power Control (DPC) algorithm $[9,8]$

$$
\bar{p}_{i}(t+1)=\bar{p}_{i}(t) \frac{\bar{\gamma}_{r e f}}{\bar{\gamma}_{i}(t)} .
$$

If the signal gains $\bar{g}_{i j}$ are constant or slowly varying, this algorithm converges to $\bar{\gamma}_{r e f}$ if this is achievable. Therefore we choose $\bar{\gamma}_{r e f} \geq \bar{\gamma}^{*}$.

The CDPC-II algorithm, proposed in [11], is more general and given by

$$
\bar{p}_{i}(t+1)=\beta \bar{p}_{i}(t)\left(\frac{1}{\bar{\gamma}_{i}(t)}\right)^{\xi} .
$$

Similar ideas are found in [14].

Analogous to the DPC algorithm in (1) is the following Constant Received Power (CRP) algorithm in [2]. In the case of unconstrained transmission powers, it is given by

$$
\bar{p}_{i}(t+1)=\bar{p}_{i}(t) \frac{\bar{C}_{t g t}}{\bar{C}_{i}(t)},
$$

where $\bar{C}_{t g t}$ is the target carrier power which the carrier power $\bar{C}_{i}=\bar{p}_{i} \bar{g}_{i i}$ strives towards. The idea of tracking a target carrier is commonly applied in narrowband CDMA systems.

As shown in [5], these algorithms are special cases of the following algorithm, in which logarithmic values are used:

$$
p_{i}(t+1)=p_{i}(t)+\beta\left(\gamma_{r e f}(t)-\gamma_{i}(t)\right)
$$

This is recognized as an integrating (I) controller. Modifications for the case of constrained powers are provided in $[2,10,5]$.

When the network is operating close to its capacity limit, the performance is degraded due to the high powers used by terminals with very bad connections. An attempt to employ graceful degradation is proposed in [1]. The distributed algorithm, which will be referred to as the AAW algorithm, is given by

$$
p_{i}(t+1)=\beta p_{i}(t)+\beta\left(\gamma_{r e f}-\gamma_{i}(t)\right) .
$$

This algorithm is further discussed in $[15,5]$.

In this paper, will concentrate on the I-controller in (4) and the AAWalgorithm in (5).

\subsection{The System Model from a Control Theory Perspective}

Define the delay operator in the time domain as

$$
q^{-1} p(t)=p(t-1) .
$$


A time delay of $n$ samples is represented by

$$
\gamma_{i}(t-n)=q^{-n} \gamma_{i}(t)
$$

Applying the delay operator to (4) yields

$$
\begin{aligned}
q p_{i}(t) & =p_{i}(t)+\beta\left(\gamma_{r e f}-\gamma_{i}(t)\right) \\
p_{i}(t) & =\frac{\beta}{q-1}\left(\gamma_{r e f}-\gamma_{i}(t)\right)
\end{aligned}
$$

The measured (or estimated) C/I:s, denoted by $\hat{\gamma}_{i}$, may be inaccurate or corrupted by noise. Therefore various filters are used to obtain smooth estimates. Two common choices are discussed here. An obvious choice is to use the average value over the $L$ last values. Using the delay operator, this Moving Average (MA) filter is given by

$$
\begin{aligned}
\gamma_{i}(t) & =\frac{1}{L}\left(\hat{\gamma}_{i}(t)+\ldots+\hat{\gamma}_{i}(t-L+1)\right)= \\
& =\frac{1}{L}\left(1+\ldots+q^{-L+1}\right) \hat{\gamma}_{i}(t)=F_{M A}(q) \hat{\gamma}_{i}(t) .
\end{aligned}
$$

In the filter above, the last $L$ values are weighted equal. As an alternative, an Exponential Forgetting (EF) filter, which exponentially forgets old values, may be used. It is defined by the recursion

$$
\gamma_{i}(t+1)=\lambda \gamma_{i}(t)+(1-\lambda) \hat{\gamma}_{i}(t+1), 0 \leq \lambda<1,
$$

or using the delay operator

$$
\gamma_{i}(t)=\frac{(1-\lambda) q}{q-\lambda} \hat{\gamma}_{i}(t)=F_{E F}(q) \hat{\gamma}_{i}(t)
$$

As a rule of thumb, the number of terms that are contributing to the output can be approximated by

$$
L_{E F} \approx \frac{1}{1-\lambda}
$$

In this paper we will provide stability analysis when using one of the smoothing filters above. However, most expressions will be derived considering the case of the I-controller and the moving average filter. Expressions for the other combinations can be derived analogously.

The distributed power control algorithm and its surroundings as in Figure 1 will be referred to as the local loop. In case of the general controller $R(q)$ and the smoothing filter $F(q)$, the local loop can be depicted as in Figure 2. The block $f(\cdot)$ describes all the constraints on the output power. If no constraints are present, this block is equal to unity and can be removed, but in the general case we have $\tilde{p}_{i}(t)=f\left(p_{i}(t)\right)$. Furthermore, the $\mathrm{C} / \mathrm{I}$ expression in $\mathrm{dB}$ is given by

$$
\hat{\gamma}_{i}(t)=\tilde{p}_{i}(t)+g_{i i}(t)-I_{i}(t)
$$




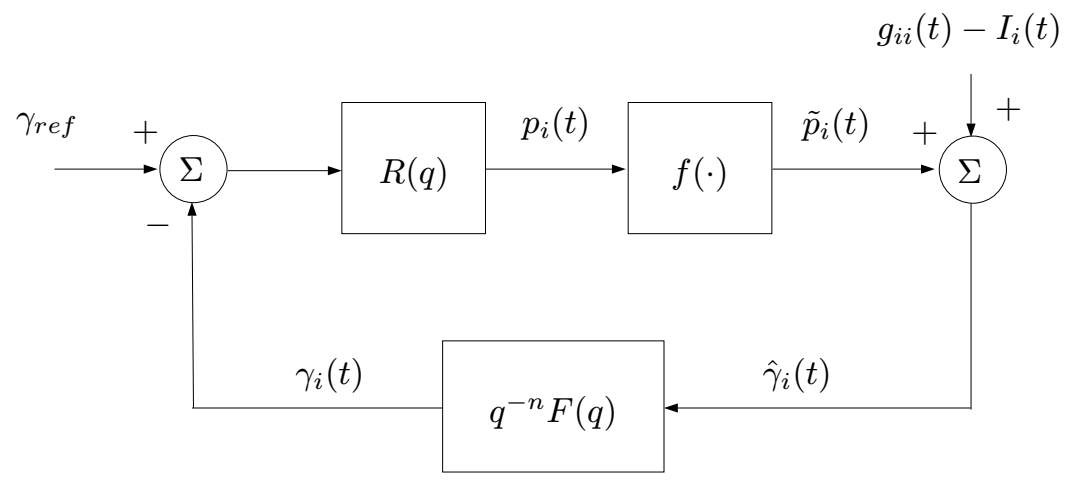

Figure 2: The local loop when using the general power control algorithm $R(q)$ and the smoothing filter $F(q)$.

The overall system contains several local loops interacting and affecting each other, since the interferences $I_{i}(t)$ are interconnected. This is referred to as the global system. When a number of stable local loops are interconnected, they affect each other in a way that is not described by an analysis of the local loop. As a matter of fact, additional dynamics might be found in the global system due to these interconnections. Therefore, it is intuitive that local stability does not guarantee global stability. However, if any of these local loops are unstable, the global system is most likely unstable as well. This is not a general conclusion, but in the case of power controlled cellular networks, the disturbance (i.e. interference) at one receiver is a strictly increasing function of the powers used by others, Therefore a "competition" between the users can be observed, and we can argue that local instabilities imply an unstable global system.

\section{Root Locus Techniques}

When neglecting the constraints, the remaining local loop is linear. Then the closed-loop system, which relates the output $\tilde{p}_{i}(t)=f\left(p_{i}(t)\right)=p_{i}(t)$ to the inputs is given by (9).

$$
p_{i}(t)=\frac{R(q)}{1+R(q) F(q)}\left(\gamma_{r e f}-F(q)\left(g_{i i}(t)-I_{i}(t)\right)\right) .
$$

To analyze stability, it is interesting to see how the closed-loop poles, i.e. the roots to the denominator polynomial, are varying with the controller parameters. 
In the case of the I-controller and the moving average filter, we have

$$
\begin{aligned}
& \frac{R(q)}{1+R(q) F(q)}=\frac{\frac{\beta}{q-1}}{1+\frac{\beta}{q-1} q^{-n} F_{M A}(q)} \\
& =\frac{\beta q^{L+n-1}}{q^{L+n}-q^{L+n-1}+\frac{\beta}{L}\left(q^{L-1}+\ldots+1\right)}
\end{aligned}
$$

We are interested in the $\beta$ :s yielding exponential stability, that is when all the denominator poles of (9) are inside the unit circle [5, 12]. The locations of the poles as functions of the parameter $\beta$ are plotted in root locus plots, see Figure 3 .
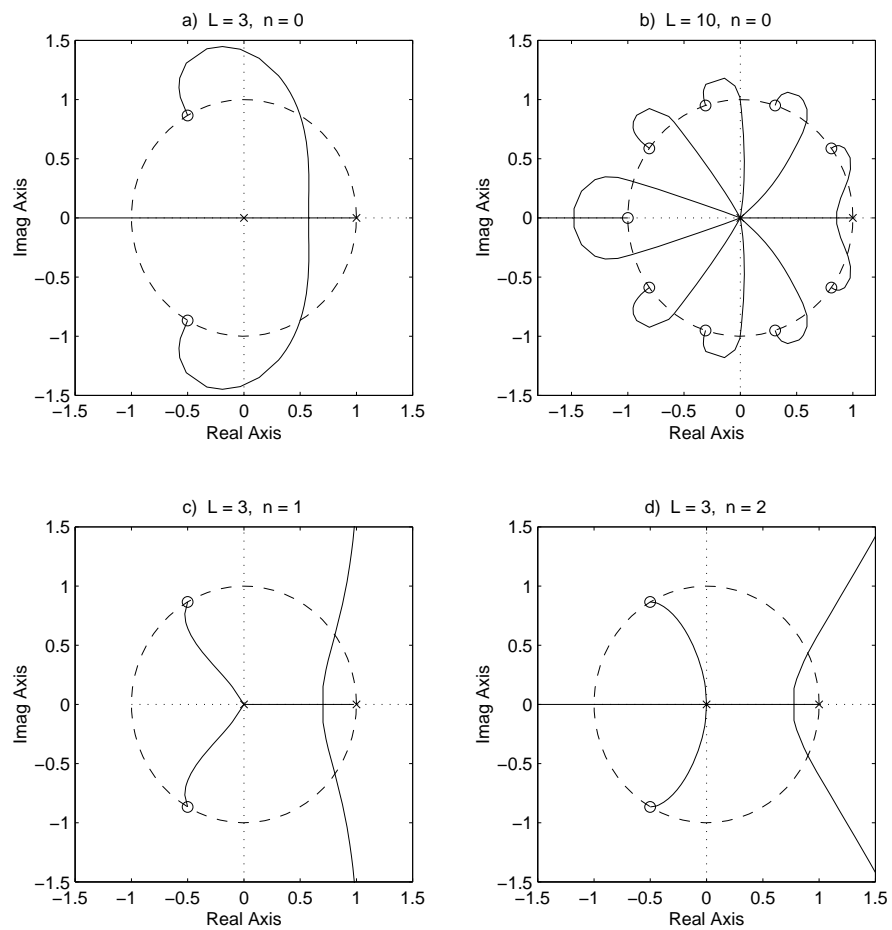

Figure 3: Root locus plots for some different window lengths $L$ and time delays $n$ when using a moving average smoothing filter and an I-controller. The ' $\mathrm{x}$ ': $\mathrm{s}$ mark the roots corresponding to $\beta=0$, while 'o' corresponds to the roots when $\beta \rightarrow \infty$.

To see the effects of time delays, the stability constraints on $\beta$ for different combinations of power control algorithms and smoothing filters are listed in Table 1 . The results yield instability limits for $\beta$ and thus larger $\beta$-values will result in an unstable system. The results indicate that the exponential forgetting filter is the better choice, yielding better performance with respect to stability. Note that in the case of a simple time delay $(L=1$ and $n=1)$ the DPC, given by the I-controller (4) and $\beta=1$, have poles located on the unit circle and gets an oscillatory behavior. Additional time delays will result in an unstable system. 


\begin{tabular}{|c||c||c|}
\hline & I-controller & AAW-algorithm \\
\hline \hline $\mathrm{n}=0$ & $>1$ & $>1$ \\
\hline $\mathrm{n}=1$ & 1.000 & 1.000 \\
\hline $\mathrm{n}=2$ & 0.618 & 0.708 \\
\hline
\end{tabular}

(a) No filter applied.

\begin{tabular}{|c||c|c||c|c|}
\hline \multicolumn{1}{|c||}{} & \multicolumn{2}{c||}{ I-controller } & \multicolumn{2}{c|}{ AAW-algorithm } \\
\cline { 2 - 5 } & MA & EF & MA & EF \\
\hline \hline $\mathrm{n}=0$ & 0.489 & $>1$ & 0.842 & $>1$ \\
\hline $\mathrm{n}=1$ & 0.353 & 1.000 & 0.759 & 1.000 \\
\hline $\mathrm{n}=2$ & 0.278 & 0.512 & 0.700 & 0.917 \\
\hline
\end{tabular}

(b) Moving average filter $(L=10)$ and exponential forgetting filter $(\lambda=0.90)$ applied.

Table 1: Local loop stability region given by upper bounds on $\beta$ using root locus techniques.

This is illuminated by the simple simulation of the local loop in Figure 4, where the effects of a step change in the interference are studied.
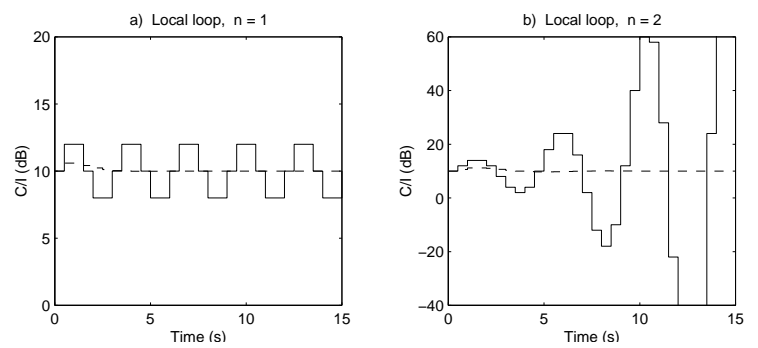

Figure 4: Simulation of an isolated local loop, when using the I-controller and no smoothing filter. The solid lines correspond to the DPC algorithm $(\beta=1)$, while the dashed to $\beta=0.3$. a) Time delay: one sample, $n=1$. b) Time Delay: two samples, $n=2$.

\section{Describing Functions in Discrete Time}

Using describing functions in discrete time one can analyze problems characterized by the block diagram in Figure 5 . The system consists of a linear part with transfer function $G(q)$, and a static non-linearity given by the function $f(\cdot)$.

We proceed by making the $N$-periodic ansatz $e(t)=C \sin (\Omega t)$, where $\Omega=$ $\frac{2 \pi}{N}$. Since $f(\cdot)$ is a static non-linearity, $w(t)$ is $N$-periodic as well. Using time discrete Fourier series expansion, the signal $w(t)$ is decomposed into its Fourier 


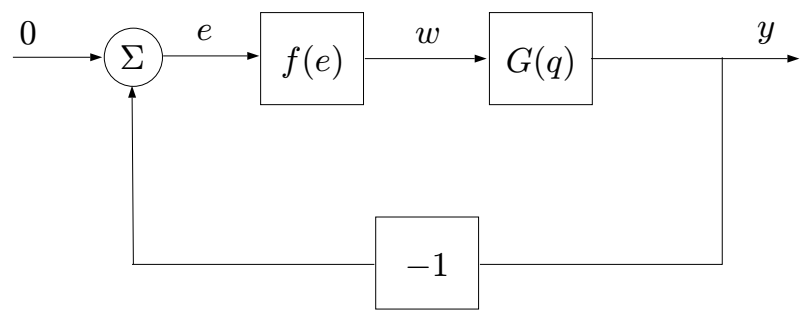

Figure 5: Block diagram of a non-linear system, separated in one linear and one non-linear part.

components

$$
\begin{aligned}
w(t) & =f(C \sin (\Omega t))=A(C, \Omega) \sin (\Omega t+\phi(C, \Omega))+ \\
& +A_{2}(C, \Omega) \sin \left(2 \Omega t+\phi_{2}(C, \Omega)\right)+\ldots
\end{aligned}
$$

where we have assumed $f(\cdot)$ to be odd for simplicity. The method, however, applies to general static functions. Furthermore assume that the linear system $G(q)$ attenuates the harmonics much more than the fundamental frequency. This is the only approximation made in the analysis. Since $e(t)=-y(t)$ according to Figure 5, we conclude that the condition for oscillations is that the loop gain is equal to -1 . The loop gain consists of an amplitude gain and a phase shift, which in turn are functions of the period $N$ and the amplitude $C$. Graphically, we can plot the loop gain in the complex plane for different $N$ and $C$ and then try to find the solution $C=C_{0}, N=N_{0}$ that yields an intersection with -1 . The solution can also be found analytically in some cases. This method is referred to as describing function analysis [4].

\section{Analysis of Systems with Non-linearities}

Generally speaking, non-linearities can be a part of our system for two reasons: Either it is a part of the power control algorithm, or it describes some constraints in the hardware, for example an upper limit on the power the transmitter can deliver. The first case is analyzed in $[5,6]$, while the second will be in focus in the following. These analyzes are based on the assumption that $g_{i i}(t)$ and $I_{i}(t)$ are varying slowly.

\subsection{The Effects of Quantization and Saturation}

In most mobile systems the available power levels form a discrete set. It is clear that this should affect the performance. Usually the power levels are regularly distributed in the $d B$ scale. The quantization and saturation can be described by replacing $f$ in Figure 2 by the blocks in Figure 6 .

Next, we would like to analyze the stability properties of the system described above. In order to do this we perturb the system, and look at its ability 


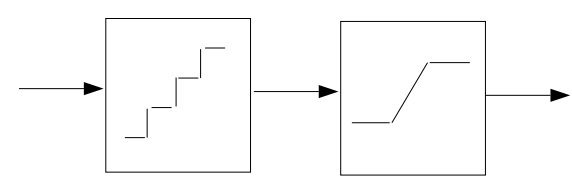

$f(\cdot)$

Figure 6: The studied constraints: Quantization followed by saturation.

to damp the error. Figure $7 \mathrm{a}, \mathrm{c}$ show how the oscillation is damped for a system with a moving average filter $(L=10)$ and $n=1$ for the values $\beta=0.3$ and $\beta=0.4$. These values correspond to stable local loops in the unconstrained case. We see that there is a fundamental difference between the asymptotic behaviors of the systems: The system with the smaller $\beta$ converges to a fixed value, while the other does not, even though both systems are well below the stability limits for the system with the non-linearities removed, see Table 1.
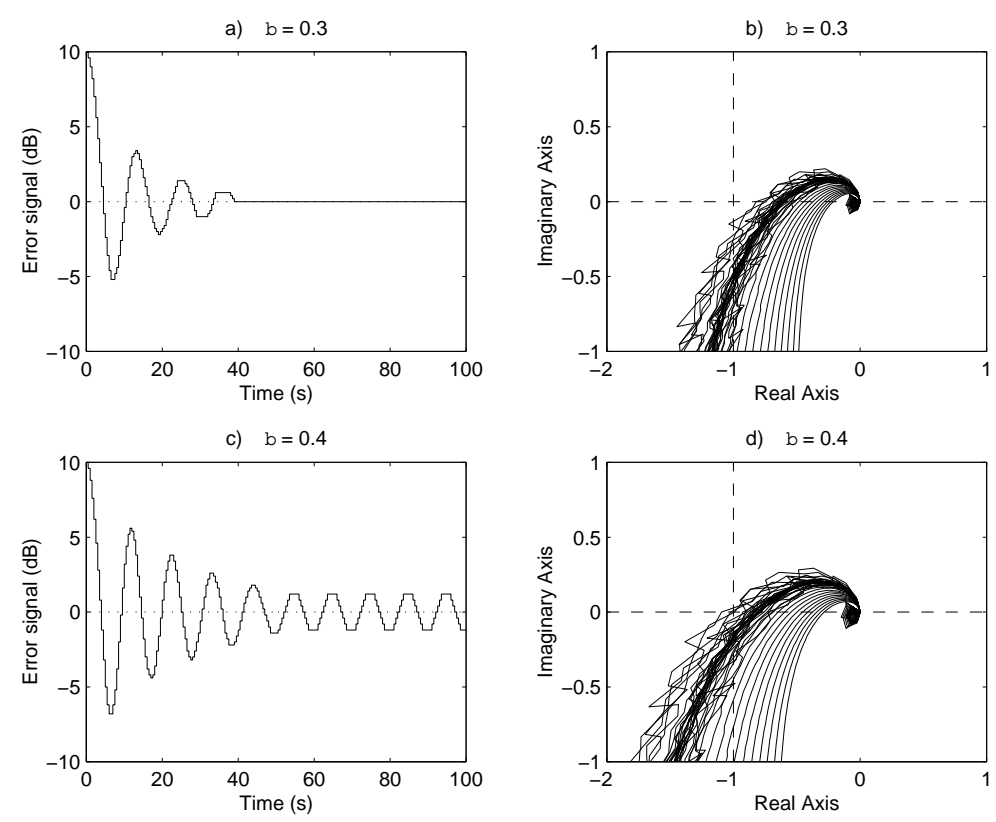

Figure 7: The effect of quantization and saturation on the error signal.

We plot the loop gain as a function of $N$ and $C$, see Figure 7b,d. Each line represent a fixed value of $C$. The method can be used to search for the smallest $\beta$ which will give an oscillation, due to the criterion that there will be an oscillation if the point -1 is covered. This search has been done for the same systems as analyzed before, and the results summarized in Table 2 should be compared to Table 1. Again, the exponential forgetting smoothing filter is the most appealing choice of filters when considering stability aspects. It is possible to use higher $\beta$-values in the controller, which corresponds to faster reactions. 


\begin{tabular}{|c||c||c|}
\hline & I-controller & AAW-algorithm \\
\hline \hline $\mathrm{n}=0$ & $>1$ & $>1$ \\
\hline $\mathrm{n}=1$ & 0.503 & 0.877 \\
\hline $\mathrm{n}=2$ & 0.431 & 0.618 \\
\hline
\end{tabular}

(a) No filter applied.

\begin{tabular}{|c||c|c||c|c|}
\hline \multicolumn{1}{|c||}{} & \multicolumn{2}{c||}{ I-controller } & \multicolumn{2}{c|}{ AAW-algorithm } \\
\cline { 2 - 5 } & MA & EF & MA & EF \\
\hline \hline $\mathrm{n}=0$ & 0.337 & $>1$ & 0.489 & $>1$ \\
\hline $\mathrm{n}=1$ & 0.247 & 0.697 & 0.353 & 0.964 \\
\hline $\mathrm{n}=2$ & 0.198 & 0.373 & 0.278 & 0.512 \\
\hline
\end{tabular}

(b) Moving average filter $(L=10)$ and exponential forgetting filter $(\lambda=0.90)$ applied.

Table 2: Limits of $\beta$ that give oscillations, obtained from describing functions analysis.

\section{Global Simulations}

As argued in Section 3 our analysis is not global, and thus the assumptions of constant interference is not valid. Consider a setting of four mobile stations with fixed locations interfering with each other. Furthermore, there is a time delay of one sample in the loop, no smoothing filter is applied, and the I-controller with different $\beta$-values is used. This case is analogous to the situation in the local loop simulation in Figure 3a.
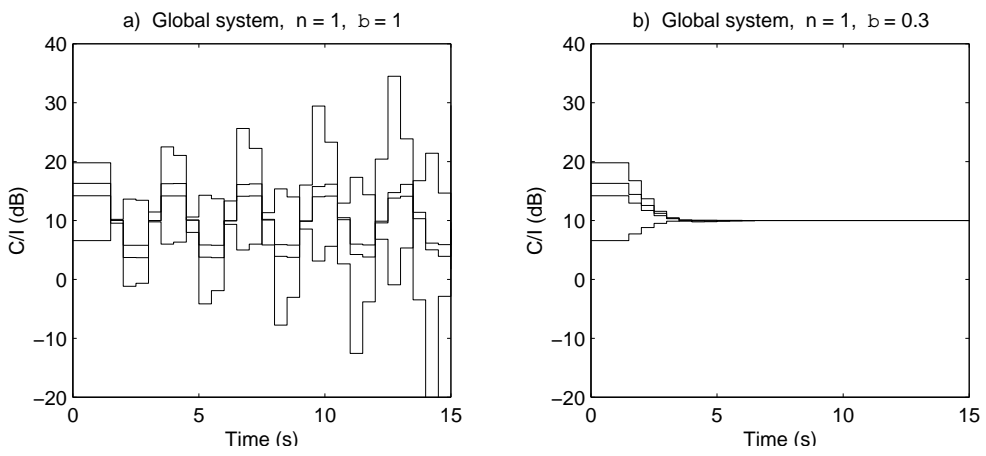

Figure 8: The effects of a time delay of a single sample. The DPC-algorithm (a) gets unstable, while the I-controller with $\beta=0.3$ (b) converges to the desired $\mathrm{C} / \mathrm{I}=10 \mathrm{~dB}$.

To study the effects of the constraints, we perform global simulations of the situation in Figure 7. Using $\beta=0.3$ in the controller we obtain the error signals as in Figure 9a. This controller was stable in the local loop, but here we see 
that the global system shows an oscillatory behavior. However these are rather limited in amplitude. A controller with $\beta=0.4$ resulted in oscillations in the local loop and as seen in Figure 9b, the global system gets unstable.
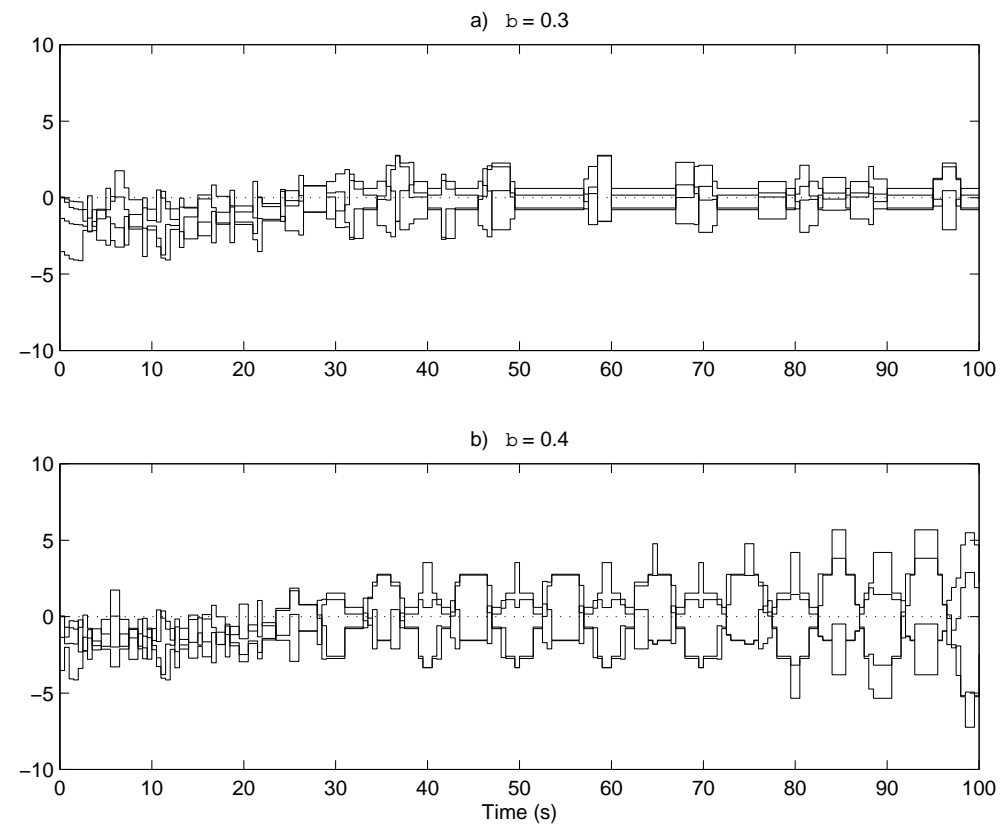

Figure 9: The error signals $e_{i}(t)=\gamma_{r e f}-\gamma_{i}(t)$ (in $\mathrm{dB}$ ) in the case when $L=10$, $n=1$ and four transmitters are interfering with each other.

\section{Conclusions}

We have studied the effects of time delays and constrained on a power controlled cellular radio networks. As the general examples we have used the I-controller, of which the DPC algorithm [8] is a special case, and the AAW-algorithm, proposed in [1]. These algorithms are relying on measurements or estimates, which may be corrupted by noise. Therefore various filters are applied to obtain smooth estimates. We have discussed the effects of using moving average or exponential forgetting filters.

Stability has been analyzed in the local power control loops, and we have argued for the extendibility of these results when considering global stability issues. Formally we say that local instability will result in global instability, but local stability does not guarantee global stability.

Time delays strongly affect the stability of the systems. Among other things it is concluded that the DPC algorithm yields an unstable global system when subject to a time delay of one sample. Constraints further hamper the stability of the systems. 
When applying filters, the analysis yields that the exponential forgetting filter results in better performance with respect to stability, than the moving average filter.

The results stress the importance of identifying the non-linearities and time delays present in the system. They are strongly affecting stability and performance of the overall system, and when known, they can be considered in the design phase using the techniques presented in this paper. 


\section{References}

[1] M. Almgren, H. Andersson, and K. Wallstedt. Power control in a cellular system. In Proc. IEEE Vehicular Technology Conference, Stockholm, Sweden, June 1994.

[2] E. Anderlind. Resource Allocation in Multi-Service Wireless Access Networks. PhD thesis, Radio Comm. Systems Lab., Royal Inst. Technology, Stockholm, Sweden, October 1997.

[3] M. Andersin, Z. Rosberg, and J. Zander. Distributed discrete power control in cellular PCS. Wireless Personal Communications, 6(3), 1998.

[4] D. P. Atherton. Nonlinear Control Engineering. Van Nostrand, 1975.

[5] J. Blom and F. Gunnarsson. Power Control in Cellular Radio Systems. Licenciate Thesis, Linköpings universitet, Sweden, June 1998. Available at http://www. control.isy.liu.se.

[6] J. Blom, F. Gunnarsson, and F. Gustafsson. Constrained power control subject to time delays. In Proc. International Conference on Telecommunications, Chalkidiki, Greece, June 1998.

[7] J. Blom, F. Gunnarsson, and F. Gustafsson. A new concept of power control in cellular systems reflecting challenges of today's systems. In Proc. IEEE Global Telecommunications Conference, Sydney, Australia, November 1998.

[8] G. J. Foschini and Z. Miljanic. A simple distributed autonomus power control algorithm and its covergence. IEEE Transactions on Vehicular Technology, 42(4), 1993.

[9] S. A. Grandhi, R. Vijayan, and D. J. Goodman. Distributed power control in cellular radio systems. IEEE Transactions on Communications, 42(2), 1994.

[10] S. A. Grandhi, J. Zander, and R. Yates. Constrained power control. Wireless Personal Communications, 2(1), 1995.

[11] T. H. Lee and J. C. Lin. A study on the distributed power control for cellular mobile systems. In Proc. IEEE Vehicular Technology Conference, Atlanta, GA, USA, April 1996.

[12] W. J. Rugh. Linear System Theory. Prentice Hall, Englewood Cliffs, NJ, USA, second edition, 1996.

[13] S. Ulukus and R. Yates. Stochastic power control for cellular radio systems. IEEE Transactions on Communications, 46(6), 1998.

[14] R. D. Yates. A framework for uplink power control in cellular radio systems. IEEE Journal on Selected Areas in Communications, 13(7), September 1995 .

[15] R. D. Yates, S. Gupta, C. Rose, and S. Sohn. Soft dropping power control. In Proc. IEEE Vehicular Technology Conference, Phoenix, AZ, USA, May 1997. 
[16] J. Zander. Distributed cochannel interference control in cellular radio systems. IEEE Transactions on Vehicular Technology, 41(3), August 1992.

[17] J. Zander. Performance of optimum transmitter power control in cellular radio systems. IEEE Transactions on Vehicular Technology, 41(1), February 1992. 